\title{
Dysphagia and nutrition: An extended scope of practice
}

\author{
L. Wright, D. Cotter and M. Hickson \\ Imperial College Healthcare NHS Trust, Department of Nutrition and Dietetics, Charing Cross Hospital, \\ London W6 $8 R F, U K$
}

Dysphagia requires identification and treatment from speech and language therapists (SLT), and since patients with dysphagia are at high risk of malnutrition ${ }^{(1)}$, it is also vital that nutrition screening is carried out, with dietitians instigating appropriate treatments. It has been demonstrated that dysphagia screening can accurately identify stroke patients presenting with dysphagia ${ }^{(2)}$ and it has been shown in America and Canada that dietitians can effectively use such screening processes to identify patients with dysphagia ${ }^{(3)}$. However, there is no work to date exploring whether dietetic skills could be transferable to SLTs. The purpose of this research was to establish whether UK dietitians and SLTs could successfully extend their scope of practice to enable each professional to safely screen and treat dysphagia holistically. Thus, the dietitian could assess and identify dysphagia and make appropriate texture modification or nil by mouth recommendations; and the SLT could screen for malnutrition and initiate suitable nutrition support, including enteral feeding.

The first part of the study was a combination of theory and practice based learning. It involved learning the others' skills through observation and being observed, as well as completing a reading list and conducting literature searches. A training pack for the dietitian and SLT was designed. Protocols for practice were developed to outline a step by step process that each profession should follow and where they were required to refer on for specialist opinion. The second part of the study aimed to determine whether each professional correctly assessed and treated 30 patients as judged by an independent assessor. The dietitian and SLT were assessed as they screened and treated each patient by an independent observer from the other profession (the dietitian was observed by a SLT and the SLT by a dietitian). The observer did not interact with the extended scope trainee, but simply observed the process and at the end verified whether they agreed with the diagnostic decisions and care plan. This study was approved by the local research ethics committee.

A total of 60 patients gave written informed consent to participate in this study; 30 for each professional. Both therapists achieved $97 \%$ agreement with the independent observer. There was only 1 patient for each therapist where disagreement occurred. The disagreements were both due to the extended practice trainee erring on the side of caution and following the prepared treatments protocols to put the patient nil by mouth until the patient was seen by the other therapist.

This data demonstrates that dietitians can acquire the skills to screen for and manage dysphagia and SLTs can acquire the skills to screen for and manage malnutrition. This two-fold approach to the management of patients with dysphagia by the SLT and dietitian has not previously been documented. This approach means that patients can be assessed and treated much more quickly. The time delay required for referral to and assessment by the other profession is eliminated. Extending this scope of practice for both professions enables earlier intervention through faster and accurate referrals and may result in improvements in nutritional status and overall health in patients with dysphagia. Patient safety is maintained through the thorough training of therapists experienced with the patient group, the requirement to demonstrate competencies, and the development of treatment protocols to follow. Any extended role should be developed around an identified need or gap in the service ${ }^{(4)}$. Further work is needed to address how the extended-scope practitioner roles in nutrition and dysphagia will benefit the patient and whether the service is sustainable.

Extending therapist skills to meet the needs of particular patients may help to improve the care given and maintain nutritional status.

1. Wright L, Cotter D, Hickson M et al. (2005) J Hum Nutr Diet 18, 213-216.

2. Huhmann M, Touger-Decker R, Bhyam-Gray L et al. (2004) Top Clin Nutr 19, 239-249.

3. Brody RA, Touger-Decker R, Von Hagen S et al. (2000) J Am Diet Assoc 100, 1029-1036.

4. British Dietetic Association (2006) Guidance Document on Extended Scope Practice. Professional Development Guidance Document. Birmingham: BDA. 